\title{
SENSE-REVERSING GENERATORS OF DISCRETE CONVERGENCE GROUPS IN THE PLANE
}

\author{
A. Hinkkanen
}

\begin{abstract}
Suppose that $g$ is a sense-reversing homeomorphism of the 2-sphere $S^{2}$ onto itself that generates a discrete convergence group of infinite order. We show that then $g$ is topologically conjugate to $\bar{z}+1$ if $g$ has one fixed point in $S^{2}$. Otherwise, $g$ has two fixed points and is known to be conjugate to $2 \bar{z}$.
\end{abstract}

\section{Introduction}

Following Gehring and Martin ([2, p. 335]), we say that a group $G$ of homeomorphisms of the two-dimensional sphere $S^{2}$ onto itself is a convergence group if every sequence of elements of $G$ contains a subsequence, say $g_{n}$, such that

(i) $g_{n} \rightarrow g$ and $g_{n}^{-1} \rightarrow g^{-1}$ uniformly on $S^{2}$, where $g$ is a homeomorphism; or

(ii) there are $x_{0}, y_{0} \in S^{2}$ (possibly $x_{0}=y_{0}$ ) such that $g_{n} \rightarrow x_{0}$ and $g_{n}^{-1} \rightarrow y_{0}$ uniformly on compact subsets of $S^{2} \backslash\left\{y_{0}\right\}$ and $S^{2} \backslash\left\{x_{0}\right\}$, respectively.

For example, a group of $K$-quasiconformal mappings, for a fixed $K \geq 1$, is a convergence group. The group $G$ is discrete if it does not contain a sequence of distinct elements tending to the identity mapping Id, and then only (ii) can occur. We allow the elements of $G$ as well as any Möbius transformations that we consider to be sense-reversing. Any group of Möbius transformations is called a Möbius group. When $g$ is a homeomorphism, we write $g^{0}=\mathrm{Id}$, and for $n \geq 1$ we set $g^{n}=g \circ g^{n-1}$ and $g^{-n}=\left(g^{-1}\right)^{n}$.

Let the convergence group $G$ be cyclic, generated by $g$. We assume that $g \neq \mathrm{Id}$. We ask if $g$ is topologically conjugate to a Möbius transformation, that is, if there is a homeomorphism $f$ of $S^{2}$ onto itself such that $f \circ g \circ f^{-1}$ is a Möbius transformation. That this is indeed the case when $G$ is nondiscrete, has recently been proved by Martin and the author [3].

Suppose that $G$ is discrete. Gehring and Martin ([2, p. 340]) showed that $g$ must be of one of the following three types:

(i) $g$ is called elliptic if $g$ has finite order;

(ii) $g$ is parabolic if $g$ has a unique fixed point $x_{0}$ and then $g^{n}(x) \rightarrow x_{0}$ as $n \rightarrow \infty$ or $n \rightarrow-\infty$;

Research partially supported by the U.S. National Science Foundation.

1980 Mathematics Subject Classification: Primary 30C60. 
(iii) $g$ is loxodromic if $g$ has exactly two fixed points $x_{1}$ and $x_{2}$ and then, say, $g^{n}(x) \rightarrow x_{1}$ and $g^{-n}(x) \rightarrow x_{2}$ as $n \rightarrow \infty$, uniformly on compact subsets of $S^{2} \backslash\left\{x_{2}\right\}$ and $S^{2} \backslash\left\{x_{1}\right\}$, respectively.

As Gehring and Martin observed in [2, p. 354-356], this conjugacy problem has been solved in many cases. A theorem due in part to Brouwer, Kerékjártó, and Eilenberg [1] shows that an elliptic generator $g$ is topologically conjugate to an orthogonal transformation of $S^{2}$ and thus to $h(z)=c z$ or $h(z)=c / \bar{z}$ where $c$ is a root of unity. Here and later, we identify $S^{2}$ with the extended complex plane $\overline{\mathbf{C}}=\mathbf{C} \cup\{\infty\}$, whenever convenient.

Kerékjártó [7] proved that a sense-preserving loxodromic $g$ is conjugate to $h(z)=2 z$, and hence to $h(z)=c z$ for any complex nonzero $c$ with $|c| \neq 1$ (cf. $[7$, p. 235]). In fact, his proof applies to sense-reversing loxodromic functions also and shows that such a function is conjugate to $h(z)=2 \bar{z}$ (cf. $[4, \mathrm{p} .366])$. If we start with the assumption that $g$ is loxodromic, we only need to use Sections 7 and 8 of [7, p. 261-262].

Kerékjártó [6] proved that a sense-preserving parabolic function $g$ is topologically conjugate to $h(z)=z+1$ and thus to $h(z)=z+b$ for any complex nonzero $b$. Sperner [9] obtained an analogous characterization of functions that are topologically conjugate to a translation. Kerékjártó's arguments in $[6$, Section 6 onwards], seem to apply to sense-preserving functions only. Thus it may be of some interest to give an explicit proof of the corresponding result for sense-reversing parabolic functions.

Theorem 1. Let the sense-reversing function $g$ generate a discrete convergence group of infinite order in $S^{2}$. If $g$ has exactly one fixed point in $S^{2}$, then $g$ is topologically conjugate to $h(z)=\bar{z}+1$.

This together with the preceding remarks yields the following consequence.

Corollary 1. Any cyclic convergence group in $S^{2}$ is topologically conjugate to a group of Möbius transformations.

We remark that Gehring and Martin ([2, Theorem 7.31, p. 356]) have given an example of a noncyclic discrete convergence group on $S^{2}$ that is not topologically conjugate to a Möbius group. A nondiscrete convergence group with the same property can be obtained by modifying their example so that the Fuchsian group acting on the unit disk is replaced by a suitable nondiscrete group, such as the group of all Möbius transformations of the unit disk onto itself.

\section{Proof of Theorem 1}

2.1. Let $g$ be as in Theorem 1. In view of Kerékjártó's result [6], we may perform a preliminary conjugation and assume that $g(\infty)=\infty$ and that $g^{2}(z)=$ $z+2$. (Here $g^{2}=g \circ g$.) We need to find a simply connected domain $U$ with $\infty \in \partial U$ such that $\bar{U} \backslash\{\infty\} \subset g(U)$. Then there is a Jordan curve $\gamma$ going 
through infinity such that one of the domains determined by $\gamma$ contains $U$ while the other one contains $\mathbf{C} \backslash g(U)$, and such that $\gamma \backslash\{\infty\} \subset g(U) \backslash \bar{U}$, so that $(g(\gamma) \backslash\{\infty\}) \cap \overline{g(U)}=\emptyset$. There is a sense-preserving homeomorphism $f$ of the strip $S=\{z: 0 \leq \operatorname{Re} z \leq 1\}$ onto the closure of that domain bounded by $\gamma$ and $g(\gamma)$ that intersects $\partial g(\bar{U})$. Since $g$ is sense-reversing, we can choose $f$ so that

$$
f(\bar{z}+1)=g(f(z))
$$

when $\operatorname{Re} z=0$. Now (2.1) and the definition $f(\infty)=\infty$ extend $f$ to a homeomorphism of $S^{2}$ onto itself such that $\left(f^{-1} \circ g \circ f\right)(z)=\bar{z}+1$, as required. It will be clear from the construction of $U$ that (2.1) indeed extends $f$ to all of $\mathbf{C}$.

To find a suitable domain $U$, we define $H_{q}=\{z \in \mathbf{C}: \operatorname{Re} z<q\}$ for real $q$ and set $V_{q}=H_{q} \cap g\left(H_{q}\right)$. Then $g\left(V_{q}\right)=g\left(H_{q}\right) \cap H_{q+2} \supset V_{q}$. We claim that for each $M>0$ there is $x_{M}<0$ such that

$$
F_{M}=\left\{x+i y: x<x_{M},|y| \leq M\right\} \subset V_{0} .
$$

Given $M>0$, define $\Omega=\{x+i y:-2 \leq x<0,|y| \leq M\}$. Then $g^{-1}(\Omega)$ is a bounded subset of $\mathbf{C}$. We write $E+b=\{z+b: z \in E\}$ for $E \subset \mathbf{C}$ and $b \in \mathbf{C}$. Since $g$ commutes with $g^{2}=z+2$, we have $g^{-1}(\Omega-2 n)=g^{-1}(\Omega)-2 n \subset H_{0}$ for $n \geq n_{0}$, say. Since $\Omega-2 n \subset H_{0}$, we have $\Omega-2 n \subset V_{0}$. Thus (2.2) holds with $x_{M}=-2 n_{0}$. It follows that $V_{0}$ is not empty and that $V_{0}$ has a unique component $D$ such that $F_{M} \subset D$ for all $M>0$.

We claim that $g(D) \supset D$. In any case $D$ is contained in some component of $g\left(V_{0}\right)$ since $D \subset V_{0} \subset g\left(V_{0}\right)$. There is $x_{0}$ such that $x_{0}-2 n \in D$ for all $n \geq 0$. Thus $g\left(x_{0}-2 n\right)=g\left(x_{0}\right)-2 n \in D$ for all large $n$. Hence $D \cap g(D) \neq \emptyset$ and so $D \subset g(D)$ since $g(D)$ is one of the components of $g\left(V_{0}\right)$.

We note that $D$, being one of the components of $\mathbf{C} \backslash\left(\partial H_{0} \cup \partial g\left(H_{0}\right)\right)$, is a Jordan domain. This is clear if $\partial H_{0}$ and $\partial g\left(H_{0}\right)$ have at most one point in common, and follows from a theorem of Kerékjártó otherwise ([5, p. 87], see also $[8$, p. 168]).

If $\bar{D} \backslash\{\infty\} \subset g(D)$, we take $U=D$. Otherwise, we obtain $U$ by modifying $D$ in essentially the same way as in the proof of the loxodromic case given by Kerékjártó in [7, p. 261-262]. However, we have to be slightly more careful since $g$ has no finite attractive fixed point that we could make use of.

We write $H$ for $H_{0}$. We have $D \subset H, \partial D \subset \partial H \cup \partial g(H)$ and $\partial g(D) \subset$ $\partial g(H) \cup \partial H_{2}$. So if $z \in \partial D \backslash \partial g(H)$, then $z \in \partial H$ and so $z \notin \partial g(D)$. Since $\partial D \subset \bar{D} \subset \overline{g(D)}$, we thus have $z \in g(D)$. Therefore $\partial D \backslash g(D) \subset \bar{H} \cap \partial g(H)$. Note that $g(H) \backslash \bar{H} \neq \emptyset$ and thus both $\partial H \cap \partial D$ and $\partial H \backslash \partial D$ are nonempty.

The subset $H \cap \partial D$ of $\partial D$ is open and consists of at most countably many disjoint open arcs. The same applies to the interior of $\partial D \cap \partial H \cap \partial g(H)$. Let all these arcs, if there are any, be numbered as $I_{i}$ for $i \geq 1$. Let $F$ be a homeomorphism of $\bar{H}$ onto $\bar{D}$ with $F(\infty)=\infty$, for example a conformal mapping. Then 
the $\operatorname{arcs} F^{-1}\left(I_{i}\right)=I_{i}^{\prime} \subset \partial H \backslash\{\infty\}$ are open and disjoint. Let $L_{i}$ be the open semicircle in $H$ joining the endpoints of $I_{i}^{\prime}$.

Note that $I_{i} \subset \partial g(H)$ and so $g\left(I_{i}\right) \subset \partial H_{2}$ for all $i$. Thus $g\left(F\left(L_{i}\right)\right)$ is an arc in $g(D) \cap H_{2}$ joining two points $a_{i}$ and $b_{i}$ that lie on $\partial H_{2}$. Now the segment $a_{i} b_{i}$ together with $g\left(F\left(L_{i}\right)\right)$ bounds a Jordan domain $\Omega_{i}$ contained in $g(D)$. Let $\gamma_{i}$ be a Jordan arc in $\Omega_{i} \backslash \overline{H_{1}}$ joining $a_{i}$ to $b_{i}$, and set $\gamma_{i}^{\prime}=g^{-1}\left(\gamma_{i}\right)$. The unbounded component $W$ of $D \backslash \bigcup_{i} \gamma_{i}^{\prime}$ is a Jordan domain. We have

$$
g(W) \supset g(D) \backslash \bigcup_{i}\left(\Omega_{i} \backslash \overline{H_{1}}\right) \supset D \supset W .
$$

Furthermore, the set $E_{1}=\partial W \backslash g(W)$ is a compact nowhere dense subset of $\partial H$ in the topology of $\overline{\mathbf{C}}$. Let $F_{1}$ be a homeomorphism of $\bar{H}$ onto $\bar{W}$ with $F_{1}(\infty)=\infty$.

For each integer $n \geq 1$, we cover $E_{1} \cap\{i y: n-1 \leq|y| \leq n\}$ by finitely many vertical open segments $J_{n i}$ of length less than $\varepsilon$, where $\varepsilon \in(0,1)$ is chosen so that $\left|g\left(F_{1}(z)\right)-g\left(F_{1}(w)\right)\right|<\frac{1}{2}$ if $z, w \in \overline{H_{0}} \backslash H_{-1}$ with $|z|,|w| \leq n+1$ and $|z-w| \leq \varepsilon$. Let $L_{n i}$ be the semicircle contained in $H_{0}$ and joining the endpoints of $J_{n i}$. The component $U_{1}$ of $H_{0} \backslash \bigcup_{n} \bigcup_{i} L_{n i}$ containing $H_{-1}$ is a Jordan domain, and $U=F_{1}\left(U_{1}\right)$ is a domain with the required properties. This completes the proof of Theorem 1.

\section{References}

[1] Eilenberg, S.: Sur les transformations périodiques de la surface du sphère. - Fund. Math. $22,1934,28-41$.

[2] Gehring, F.W., and G.J. Martin: Discrete quasiconformal groups I. - Proc. London Math. Soc. (3) 55, 1987, 331-358.

[3] Hinkkanen, A., and G.J. Martin: Abelian nondiscrete convergence groups in the plane. - Preprint.

[4] Homma, T., and S. Kinoshita: On the regularity of homeomorphisms of $E^{n}$. - J. Math. Soc. Japan 5, 1953, 365-371.

[5] Von KERÉKJÁRTó, B.: Vorlesungen über Topologie I: Flächentopologie. - Springer-Verlag, Berlin, 1923.

[6] Von KerÉKJÁRTó, B.: Über die fixpunktfreien Abbildungen der Ebene. - Acta Litt. Sci. Szeged 6, 1934, 226-234.

[7] Von KerÉKJÁRTó, B.: Topologische Charakterisierung der linearen Abbildungen. - Acta Litt. Sci. Szeged 6, 1934, 235-262.

[8] Newman, M.H.A.: Elements of the topology of plane sets of points. - Cambridge Univ. Press, Cambridge, 1964.

[9] Sperner, E.: Über die fixpunktfreien Abbildungen der Ebene. - Abh. Math. Sem. Hamburg 10, 1934, 1-47.

University of Illinois at Urbana-Champaign

Department of Mathematics

Urbana, Illinois 61801

U.S.A.

Received 20 July 1990 